Publ. Mat. 58 (2014), 401-414

DOI: 10.5565 /PUBLMAT_58214_20

\title{
ON SEPARATED CARLESON SEQUENCES IN THE UNIT DISC
}

\author{
ERIC AMAR
}

\begin{abstract}
The interpolating sequences $S$ for $H^{\infty}(\mathbb{D})$, the bounded holomorphic functions in the unit disc $\mathbb{D}$ of the complex plane $\mathbb{C}$, were characterized by L. Carleson using metric conditions on $S$. Alternatively, to characterize interpolating sequences we can use the existence in $H^{\infty}(\mathbb{D})$ of an infinity of functions $\left\{\rho_{a}\right\}_{a \in S}$, uniformly bounded in $\mathbb{D}$, the function $\rho_{a}$ being 1 at the point $a \in S$ and 0 at any $b \in S \backslash$ $\{a\}$. A. Hartmann recently proved that just one function in $H^{\infty}(\mathbb{D})$ was enough to characterize interpolating sequences for $H^{\infty}(\mathbb{D})$. In this work we use the "hard" part of Carleson's proof of the corona theorem to extend Hartmann's result and to answer a question he asked in his paper.
\end{abstract}

2010 Mathematics Subject Classification: 30H10, 30H80.

Key words: Interpolating sequences, Carleson measures.

\section{Introduction}

Let $\mathbb{D}$ be the unit disc in $\mathbb{C}$ and $S$ a sequence of points in $\mathbb{D}$. Let $d_{P}(a, b):=\left|\frac{a-b}{1-\bar{a} b}\right|$ be the pseudohyperbolic distance and $d_{H}(a, b):=$ $\tanh ^{-1}\left(d_{P}(a, b)\right)$ the hyperbolic distance in $\mathbb{D}$.

To say that the sequence $S$ is separated means that there is an $\eta>0$ such that

$$
\forall a, b \in S, a \neq b, d_{H}(a, b) \geq \eta \Longleftrightarrow d_{P}(a, b) \geq \tanh \eta .
$$

Equivalently, a sequence $S$ is $\delta$-separated when the discs $D(a, \delta(1-|a|))$, $a \in S$, are disjoint.

We shall also need the notion of Carleson measure. Let $(\zeta, h) \in$ $\mathbb{T} \times(0,1)$, and denote by

$$
W(\zeta, h):=\{z \in \mathbb{D} \text { s.t. }|1-\bar{\zeta} z|<h\}
$$

the associated Carleson window. If $\nu$ is a Borel measure on $\mathbb{D}$, we shall say that $\nu$ is Carleson if there is a constant $C>0$ such that

$$
\forall \zeta \in \mathbb{T}, \forall h \in(0,1),|\nu|(W(\zeta, h)) \leq C h .
$$


Definition 1.1. A sequence $S$ is called a Carleson sequence if the canonical measure associated with it

$$
\mu_{S}:=\sum_{a \in S}(1-|a|) \delta_{a}
$$

is a Carleson measure.

Definition 1.2. We say that $S$ is interpolating for $H^{\infty}(\mathbb{D})$ if for any $\lambda$ in $\ell^{\infty}(S)$ there is an $f$ in $H^{\infty}(\mathbb{D})$ such that for any $a$ in $S$ we have $f(a)=\lambda_{a}$.

L. Carleson [1] characterized these sequences by the condition:

$$
\inf _{a \in S} \prod_{b \in S \backslash\{a\}}\left|\frac{a-b}{1-\bar{b} a}\right|>0 .
$$

It is easily seen that this condition is equivalent to the fact that $S$ is dual bounded in $H^{\infty}(\mathbb{D})$, (or weakly interpolating) which means:

$\exists C>0, \forall a \in S, \exists \rho_{a} \in H^{\infty}(\mathbb{D}),\left\|\rho_{a}\right\|_{\infty} \leq C$ s.t. $\forall b \in S, \rho_{a}(b)=\delta_{a b}$

To see this, it is enough to take $\rho_{b}(z):=\frac{B_{b}(z)}{B_{b}(b)}$ with $B_{b}(z):=\prod_{a \in S \backslash\{b\}} \frac{a-z}{1-\bar{a} z} \frac{|a|}{a}$.

So the metric condition $(\mathrm{C})$ which characterizes interpolation is equivalent to the functional characterization, namely the existence of an infinity of functions satisfying the above conditions.

Another functional characterization is due to D. Sarroste [5]:

Theorem 1.3. If there is $0<\tau<\eta$ such that for any partition $A, B$ of $S$, i.e. $S=A \uplus B$, there is a function $f \in H^{\infty}(\mathbb{D}),\|f\|_{\infty} \leq 1$, such that for any $a$ in $A,|f(a)| \leq \tau$ and for any $b$ in $B,|f(b)| \geq \eta$, then $S$ is $H^{\infty}(\mathbb{D})$-interpolating.

Again it requires an infinity of functions in $H^{\infty}(\mathbb{D})$ to characterize interpolating sequences.

A. Hartmann [3] showed that this can be reduced to a condition on only one function:

Theorem 1.4. Let $S$ be a separated Blaschke sequence in the unit disc $\mathbb{D}$ of $\mathbb{C}$. There is a partition $(A, B)$ of $S$ such that if there is a function $f \in$ $H^{\infty}(\mathbb{D})$ with $f=0$ on $A$ and $f=1$ on $B$, then $S$ is interpolating for $H^{\infty}(\mathbb{D})$. 
A natural question after these results is: is it possible to have an analogous result as D. Sarroste replacing for any partition by there is a partition?

The aim of this work is to prove that the answer is yes, provided that $\tau<\eta^{\kappa}$ for a certain constant $\kappa>1$ introduced by Carleson in his proof of the corona theorem.

\subsection{Good partitions.}

We need the following notions.

Definition 1.5. A partition $(A, B)$ of a sequence of points $S \subset \mathbb{D}$ is "good" if there is $\varphi: A \rightarrow B$ such that $\forall a \in A, d_{H}(a, \varphi(a))=$ $\inf _{c \in S \backslash\{a\}} d_{H}(a, c)$ and if there is $\psi: B \rightarrow A$ such that $\forall b \in B$, $d_{H}(b, \psi(b))=\inf _{c \in S \backslash\{b\}} d_{H}(b, c)$.

1.1.1. Restricted good partition. We shall need more specific partitions of a discrete sequence. Let $\gamma \in] 0,1[$; we set

$$
C_{n}=C_{n}(\gamma):=\left\{z \in \mathbb{D}: 1-\gamma^{n}<|z| \leq 1-\gamma^{n+1}\right\} .
$$

Let $S$ be a discrete sequence in the disc, let $\gamma \in] 0,1[$, and for any partition $(A, B)$ of $S$, set $A_{n}:=A \cap C_{n}(\gamma), B_{n}:=B \cap C_{n}(\gamma), S_{n}:=S \cap C_{n}(\gamma)$.

Definition 1.6. A restricted good partition of the discrete sequence $S$ in the disc is a partition $(A, B)$ of $S$ such that there is a $\gamma$ in $] 0,1[$ so that $\left(A_{n}, B_{n}\right)$ is a good partition of $S_{n}$ for any $n \in \mathbb{N}$.

As we shall see later a discrete sequence $S$ in the disc always admits a restricted good partition.

1.1.2. Hoffman partition. Let $S$ be a discrete sequence in the disc. We shall cut $S$ in two parts; for this let

$$
D_{1}:=\left\{z \in \mathbb{D}: \operatorname{Arg} z \in\left[0, \pi[\}, \quad D_{2}:=\{z \in \mathbb{D}: \operatorname{Arg} z \in[\pi, 2 \pi[\} .\right.\right.
$$

Now set $S_{1}:=S \cap D_{1}, S_{2}:=S \cap D_{2}$. Since the union of two Carleson sequences is also a Carleson sequence, it will be enough to prove that $S_{1}$ and $S_{2}$ are Carleson to get that $S$ is Carleson. So let $S=S_{1}$.

For convenience we shall work in the upper half plane $\mathbb{C}^{+}$. We can assume that $\forall a \in S, 0 \leq \Re a \leq L<\infty, 0<\Im a \leq H<\infty$ and $C_{n}=C_{n}(\gamma):=\left\{z \in \mathbb{C}^{+}: \gamma^{n+1}<\Im z \leq \gamma^{n}\right\}, \gamma$ being fixed later.

We start with the point $a_{0}=x_{0}+i y_{0}$ in $S_{n}:=C_{n} \cap S$ with the biggest $y_{0}$ among the points with the smallest real part $x_{0}$; if $\# S_{n}=1$, put $a_{0}$ in $A_{n}$ and set $B_{n}:=\emptyset$; if not, take the next point $b=x+i y$ in $S_{n}$ with the same real part as $a_{0}$ hence with a smaller imaginary part, 
if any, or such that its real part $x$ is as small as possible; if there is more than one such point, choose the one with biggest $y$. Call this point $b_{0}$ and define $\varphi\left(a_{0}\right):=b_{0}$. This implies in particular that if $\Re b_{0}=\Re a_{0}$ then there is no point of $S_{n}$ in the vertical segment $\left(a_{0}, b_{0}\right)$; if $\Re b_{0}>\Re a_{0}$ then there is no point of $S_{n}$ in the strip $\left\{z \in C_{n}: \Re a_{0} \leq \Re z<\Re b_{0}\right\}$. If $S$, hence $S_{n}$, is $\delta$-separated and if $\gamma>\frac{1}{1+2 \delta}$, then the only possibility is $\Re b_{0}>\Re a_{0}$. To see this recall that $d_{P}(a, b)=\left|\frac{a-b}{a-b}\right|$. Hence, if $\Re a=\Re b$ then $d_{P}(a, b)=\left|\frac{\Im a-\Im b}{\Im a+\Im b}\right| \geq \delta$. But if $a, b \in C_{n}(\gamma)$ then $|\Im a-\Im b| \leq$ $\gamma^{n}-\gamma^{n+1}=\gamma^{n}(1-\gamma)$ and $|\Im a+\Im b| \geq 2 \gamma^{n+1}$ which implies

$$
\delta \leq\left|\frac{\Im a-\Im b}{\Im a+\Im b}\right| \leq \frac{1-\gamma}{2 \gamma} \Rightarrow \gamma \leq \frac{1}{1+2 \delta} .
$$

So if $\gamma>\frac{1}{1+2 \delta}$, then $a$ and $b$ cannot be in the same $C_{n}(\gamma)$.

Now take the next point in $S_{n}$ at the right side of $b_{0}$, i.e. the same way as above, and call it $a_{1}$, etc. Then each time define $\varphi\left(a_{j}\right):=b_{j}$. Call $A_{n}$ the set of all $a_{j}$ 's and $B_{n}$ the set of all $b_{j}$ 's. We have that $A_{n}$ and $B_{n}$ are finite because $\forall a \in S, 0 \leq \Re a \leq L<\infty, 0<\Im a \leq H<\infty$. So by construction we always have that if $\Re b_{j}=\Re a_{j}$ then there is no point of $S_{n}$ in the vertical segment $\left(a_{j}, b_{j}\right)$; if $\Re b_{j}>\Re a_{j}$ then there is no point of $S_{n}$ in the strip $\left\{z \in C_{n}: \Re a_{j} \leq \Re z<\Re b_{j}\right\}$. Again if $S$, hence $S_{n}$, is $\delta$-separated and if $\gamma>\frac{1}{1+2 \delta}$, then the only possibility is $\Re b_{j}>\Re a_{j}$, which means that the points of $S_{n}$ are disposed on one "horizontal file". See [2, Hoffman's theorem, p. 402].

Definition 1.7. Let $A:=\bigcup_{n \in \mathbb{N}} A_{n}$ and $B:=\bigcup_{n \in \mathbb{N}} B_{n}$ then $(A, B)$ is the Hoffman partition of $S$ associated to $\gamma \in] 0,1[$.

Definition 1.8. Let $(A, B)$ be a restricted good partition or a Hoffman partition of the sequence $S \subset \mathbb{D}$. Let $\kappa \geq 1$ be a constant, the sequence $S \subset \mathbb{D}$ is $\kappa$-ultra-separated if $S$ is separated and if there are $0<\tau<\eta<1, \tau<\eta^{\kappa}$, and a function $f$ in $H^{\infty}(\mathbb{D}),\|f\|_{\infty} \leq 1$, such that $|f| \leq \tau$ on $A$ and $|f| \geq \eta$ on $B$.

Now we can state the theorem.

Theorem 1.9. There is a constant $\kappa>1$ such that the sequence $S$ is $H^{\infty}(\mathbb{D})$-interpolating if and only if it is $\kappa$-ultra-separated.

This constant $\kappa$ was introduced by Carleson in his proof of the corona theorem. 
Theorem 1.9 generalizes the result of A. Hartmann and answers positively his question:

If there is an $f$ in $H^{\infty}(\mathbb{D})$ such that $f(a)=0$, for any $a$ in $A$ and $|f(b)| \geq \eta>0$ for any $b$ in $B$, where $(A, B)$ is a Hoffman partition of $S$, and if $S$ is separated, is $S$ interpolating?

I introduce also "good" partitions for dealing with this problem in the unit ball of $\mathbb{C}^{n}$. This notion is invariant by automorphisms and hence more natural that the Hoffman partition. The result in the ball, not as good as in the disc, will be posted later. It involves complex geometry and the key fact used is that the measure $(1-|z|)|\partial f(z)|^{2} d m(z)$ is a Carleson measure in the unit ball of $\mathbb{C}^{n}$.

\section{General facts}

Lemma 2.1. Let $S$ be a discrete sequence in the metric space $(X, d)$. Then there is a good partition $\left(S_{1}, S_{2}\right)$ of $S$.

Proof: Take a point $O \in X$ and $a_{1} \in S$ such that $d\left(a_{1}, O\right)$ is minimal, if $\# S=1$ set $\varphi\left(a_{1}\right)=a_{1}$ and $S_{1}:=\left\{a_{1}\right\}=S ; S_{2}=\emptyset$; then we are done.

If $\# S \geq 2$, then take $b_{1} \in S$ a nearest neighbour for the distance $d$ of $a_{1}$ and define $\varphi\left(a_{1}\right)=b_{1}$. By the assumption on the cardinality of $S, b_{1}$ exists. Take $a_{2}$ a nearest neighbour of $b_{1}$, if it exists, and define $\psi\left(b_{1}\right):=a_{2}$; if $a_{2}=a_{1}$ we stop at this "perfect" pair $\left(a_{1}, b_{1}\right)$ with $\psi\left(b_{1}\right):=a_{1}$. If not we continue with $b_{2}$ a nearest neighbour of $a_{2}$, etc. We stop at a perfect pair. This way we get a branch $B_{1}$ finite or infinite. We put all the " $a$ " in $S_{1}$ and all the " $b$ " in $S_{2}$.

If it remains any point in $S$, then we have that the points in $S \backslash B_{1}$ are far from the points in $B_{1}$ by construction. We take a point $c$ in $S \backslash B_{1}$ which is the nearest from $O$.

A) If all the nearest points from $c$ are in $B_{1}$, which may happen, then we take one of them, $d$. Now if $d$ is in $S_{1}$, then we put $c$ in $S_{2}$ and we set $\psi(c):=d$. If $d$ is in $S_{2}$, then we put $c$ in $S_{1}$ and we set $\varphi(c):=d$. This completes $B_{1}$ and we start again.

B) If $c$ has a nearest neighbour which is not in $B_{1}$, then we start a new branch $B_{2}$. A new point may have its nearest neighbour in $B_{1}$ or in $B_{2}$, then we put it in $B_{1}$ or in $B_{2}$, as in the step A.

We continue this way in order to exhaust $S$. The $S_{1}$ part is all the "a" and $S_{2}$ is all the "b". Then $S$ is a bipartite graph with components $S_{j}$, $j=1,2$ on which the two mappings $\varphi, \psi$ are well defined. 


\section{Proof of the main theorem}

Let $S$ be a discrete sequence in the unit disc $\mathbb{D}$. Fix any $\gamma$ in $] 0,1[$ and $n \in \mathbb{N}$ and recall

$$
C_{n}=C_{n}(\gamma):=\left\{z \in \mathbb{D} \text { s.t. } 1-\gamma^{n}<|z| \leq 1-\gamma^{n+1}\right\}, \quad S_{n}:=S \cap C_{n} .
$$

We shall use the following lemmas.

Lemma 3.1. If the number of points in $S_{n}$ is smaller than a fixed number $m$ for any $n \in \mathbb{N}$, then $S$ is a Carleson sequence.

Proof: For $S$ to be Carleson it must exist a constant $C>0$ such that for any Carleson window $W=W(\zeta, h)$, we have $\sum_{a \in S \cap W}(1-|a|) \leq C h$.

If $a \in W$ then we have $1-|a| \leq h$, hence

$$
\sum_{a \in S \cap W}(1-|a|) \leq \sum_{a \in S, 1-|a| \leq h}(1-|a|) .
$$

But $a \in C_{n}(\gamma)$ implies $\gamma^{n+1}<1-|a| \leq \gamma^{n}$ and, because there are at most $m$ points in $S \cap C_{n}(\gamma)$, we have

$$
\begin{aligned}
\sum_{a \in S, 1-|a| \leq h}(1-|a|) & =\sum_{n \in \mathbb{N}, \gamma^{n+1}<h} \sum_{a \in C_{n}(\gamma) \cap S}(1-|a|) \\
& \leq m \sum_{n \in \mathbb{N}, \gamma^{n+1}<h} \gamma^{n} \leq \frac{m}{\gamma(1-\gamma)} h .
\end{aligned}
$$

Hence we have the lemma with $C=m / \gamma(1-\gamma)$.

Lemma 3.2. Let $S$ be a discrete sequence in $\mathbb{D}$, then there is a restricted good partition for $S$.

Proof: Take any $\gamma \in] 0,1\left[\right.$; because $S$ is discrete, $S_{n}:=S \cap C_{n}(\gamma)$ has only a finite number of points. If $S_{n}=\emptyset$, we simply set $A_{n}=B_{n}:=\emptyset$. If its cardinal is bigger than one, then we can apply the general Lemma 2.1: there is a good partition $\left(A_{n}, B_{n}\right)$ of $S_{n}$.

Setting $A=\bigcup_{n \in \mathbb{N}} A_{n}, B=\bigcup_{n \in \mathbb{N}} B_{n}$, we have that $(A, B)$ is a restricted good partition of $S$.

We remark that $\gamma \leq \frac{1-|a|}{1-|\varphi(a)|} \leq 1 / \gamma$ because $a$ and $\varphi(a)$ belong to the same $C_{n}(\gamma)$.

Back to the proof of the main Theorem 1.9. We suppose first that $S$ is $\kappa$-ultra-separated and we want to show that $S$ is interpolating. 
It is enough to show that $S$ is Carleson because $S$ being separated, it will be $H^{\infty}(\mathbb{D})$-interpolating.

Let $W=W(\zeta, h)$ be a Carleson window we have to show that

$$
\sum_{a \in A \cap W}(1-|a|) \leq C h .
$$

We shall cut the set $A \cap W$ in two parts.

\subsection{The points $E_{W}:=\{a \in W \cap S$ such that $\varphi(a) \notin W\}$.}

3.1.1. Case of Hoffman partition. For convenience we shall work in the upper half plane $\mathbb{C}^{+}$. Let $C_{a}$ be the strip $C_{n}(\gamma)=\left\{z: \gamma^{n+1}<\right.$ $\left.\Im z \leq \gamma^{n}\right\}$ which contains $a$. If we deal with a Hoffman partition, the point $b:=\varphi(a)$ is the nearest point in $S \cap C_{a}$, either with the same real part, hence with a smaller imaginary part, or on the right of $a$ i.e. with a bigger real part; hence if $b \notin W$ this means that there is no points of $A$ between $a$ and the right vertical side of $W$ in the strip $C_{n}(\gamma)$ containing $a$. So $a$ is the nearest point in $A$ to the right side of $W$ in the strip $C_{a}$. We shall take the maximum possible of these points, which means that we have at most one point in each $C_{n}(\gamma) \cap W$ and then we have $\sum_{a \in E_{W}} \Im a \leq C h$ by Lemma 3.1 .

3.1.2. Case of restricted good partition. We shall work again in the upper half plane. So $W$ is a square with one side on the real axis. Let $c$ be the orthogonal projection of $a$ on the side of $W$ in the direction of $b$.

We define the border strip to be a tube $T(a, r)$ around the segment $[a, c]$ of width $r \Im a$.

The partition $(A, B)$ being restricted, this means that $b$ belongs to the same $\operatorname{strip} C_{n}(\gamma):=\left\{z \in \mathbb{C}^{+}: \gamma^{n+1}<\Im z \leq \gamma^{n}\right\}$ as $a$.

Lemma 3.3. Let $(A, B)$ be a restricted good partition of $S$ in $\mathbb{D}$. Let $W=W(\zeta, h)$ be a Carleson window and $a \in A$ and $b:=\varphi(a)$ be such that $a \in W, b \notin W$. Then the border strip $T(a, r)$ contains at most a fixed number $m=m(\gamma, \delta, r)$ of points of $A$.

Proof: Because $b$ is the nearest point to $a$ in $S \cap C_{a}$ we have that there is no point of $S \cap C_{a}$ in the hyperbolic ball $Q(a, b)$ "centered" at $a$ and passing through $b$, i.e. $Q(a, b):=\left\{z \in \mathbb{C}^{+}:\left|\frac{z-a}{z-\bar{a}}\right|=\left|\frac{b-a}{b-\bar{a}}\right|\right\}$. So the worst case is when $b$ belongs to one of the three sides of $W$ in $\mathbb{C}^{+}$. Suppose first that $b$ is in the vertical left side of $W$. 
The border strip is

$$
T(a, r):=\{z=x+i y \in W \text { s.t. }(1-r) \Im a<y<(1+r) \Im a, x<\Re a\} .
$$

See Figure 1.

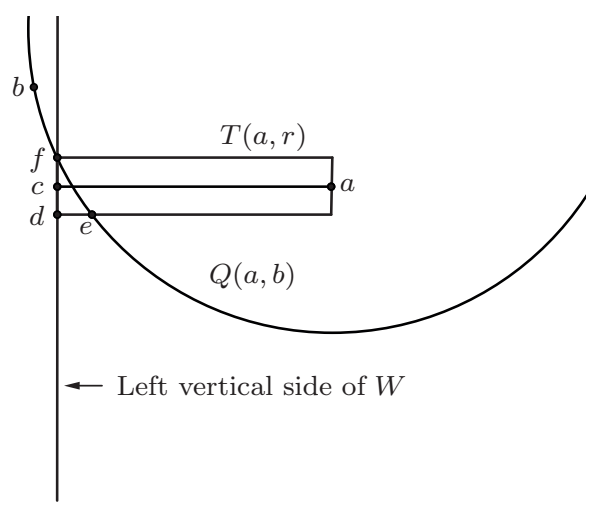

FiguRe 1.

Then the points of $A \backslash\{a\}$ in $T(a, r)$ must lie in the triangle $f d e \cap$ $T(a, r)$, but $|d e| \leq|f d| \leq \gamma^{n}(1-\gamma)$ and in this triangle there are at most $m=m(\gamma, \delta, r)$ points in $A$, because $A$ is a $\delta$-separated sequence. To see this let $p \in A \cap b d e \cap T(a, r)$, then the discs $D(p, \delta \Im p)$ are disjoint, hence the sum of their area is smaller than the area of the triangle $f d e \cap T(a, r)$. This means

$$
\sum_{p \in A \cap f \operatorname{den} T(a, r)} \pi \delta^{2}(\Im p)^{2} \leq 4 r^{2} \gamma^{2 n}
$$

But $p \in A \cap b \operatorname{de} \cap T(a, r)$ implies $\Im p \geq \Im a-r \Im a \geq(1-r) \gamma^{n+1}$ because the width of $T(a, r)$ is $r \Im a$ and $\Im a \geq \gamma^{n+1}$. So we get

$$
m \pi \delta^{2}(1-r)^{2} \gamma^{2 n+2} \leq 4 r^{2} \gamma^{2 n} \Rightarrow m \leq \frac{1}{\pi \delta^{2}(1-r)^{2}} \frac{4 r^{2}}{\gamma^{2}} .
$$

If $b$ is on the right side of $W$, this is the same.

Suppose now that $b$ is on the top of $W$. Because $b$ is the nearest point in $S \cap C_{a}$ to $a$, the points in $(A \backslash\{a\}) \cap T(a, r) \cap W$ must be in one of the triangles def or $d^{\prime} e^{\prime} f^{\prime}$ of sides less than $r \Im a \leq r \gamma^{n}$. 


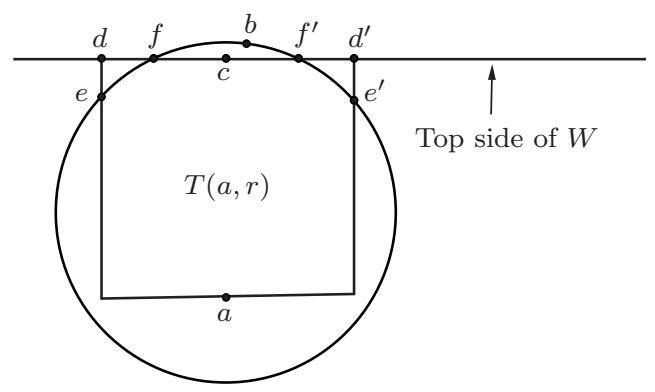

FiguRE 2.

Because $S$ is $\delta$-separated, exactly as in the previous case, there are at most $m=m(\gamma, \delta, r)$ such points. See Figure 2 .

Taking border strips $T(a, r)$ with half of the width, $r \rightarrow r / 2$, they become disjoint. Now set $E_{W}^{\prime}$ the points in $E_{W}$ such that the $T(a, r)$ is based on the vertical sides of $W$ and $E_{W}^{\prime \prime}$ the points in $E_{W}$ such that the $T(a)$ is based on the top of $W$.

We have, by Lemma 3.1, that $\sum_{a \in E_{W}^{\prime}} \Im a \lesssim h$.

For $E_{W}^{\prime \prime}$ we have that $a \in E_{W}^{\prime \prime}$ must belong to the $C_{n}(\gamma)$ with the smallest $n$ such that $W \cap C_{n}(\gamma) \neq \emptyset$. So we have $\gamma^{n} \leq \Im a \leq \gamma^{n-1}$, hence, because the tube $T(a, r)$ has width $r \Im a$, we have at most $h / r \gamma^{n}$ such tubes. So finally

$$
\sum_{a \in E_{W}^{\prime \prime}} \Im a \leq \gamma^{n-1} \frac{h}{r \gamma^{n}} \leq \frac{1}{r \gamma} h .
$$

So adding these two inequalities we get

$$
\sum_{a \in E_{W}} \Im a \lesssim h
$$

and the right estimate for $E_{W}$.

\subsection{The points $F_{W}:=\{a \in W \cap S$ such that $\varphi(a) \in W\}$.}

We shall go back to the unit disc and in this part, we shall use the "hard" part of the proof of L. Carleson of the corona theorem, as interpreted by Hörmander [4, Lemma 11, p. 948]: 
Lemma 3.4. There exists a constant $\kappa$ such that if $0<\eta<\frac{1}{2}$ and $f \in H^{\infty}(\mathbb{D})$, sup $|f| \leq 1$, then one can find $\psi$ with $0 \leq \psi \leq 1$ so that $\frac{\partial \psi}{\partial \bar{z}} d m$ is a Carleson measure in $\mathbb{D}$ and

$$
\psi(z)=0 \text { when }|f(z)|<\eta^{\kappa}, \quad \psi(z)=1 \text { when }|f(z)| \geq \eta .
$$

We shall call this $\kappa$ the Carleson constant. Because $\psi$ is real valued we also have $\frac{\partial \psi}{\partial z} d m$ is Carleson hence $|\operatorname{grad} \psi| d m$ is Carleson.

We shall use the following well known facts:

1. If $f \in H^{\infty}(\mathbb{D})$ and $|f(a)| \leq \tau$, then for $\tau^{\prime}>\tau$ there is an $r>0$ depending only on $f$ and $\tau^{\prime}$ such that:

$$
\forall z \in D(a, r(1-|a|)),|f(z)|<\tau^{\prime} .
$$

2. If $f \in H^{\infty}(\mathbb{D})$ and $|f(b)| \geq \eta$, then for $\eta^{\prime}<\eta$ there is an $r>0$ depending only on $f$ and $\eta^{\prime}$ such that:

$$
\forall z \in D(b, r(1-|b|)),|f(z)|>\eta^{\prime} .
$$

Let $(A, B)$ be the restricted or Hoffman partition of $S$ associated to the function $f \in H^{\infty}(\mathbb{D})$. Taking eventually a power of $f$, we can assume that $\eta<1 / 2$ to fit with the hypotheses of Hörmander's lemma. We have

$$
\forall a \in A,|f(a)| \leq \tau<\eta^{\kappa}, \quad \forall b \in B,|f(b)| \geq \eta .
$$

By fact 1 we have

$$
\forall a \in A, \forall z \in D\left(a, r_{1}(1-|a|)\right),|f(z)|<\tau^{\prime}
$$

and by fact 2

$$
\forall b \in B, \forall z \in D\left(b, r_{2}(1-|b|)\right),|f(z)|>\eta^{\prime} .
$$

Take $r=\min \left(r_{1}, r_{2}\right)$ to have both.

We shall need the following notions. Let $0<\alpha<1, \beta>0, a, b \in \mathbb{D}$ and set $R(a, b, \alpha, \beta)$ a tube around a smooth curve $\Gamma(a, b)$ with thickness $\tau:=\alpha \min (1-|a|, 1-|b|)$ i.e.

$$
R(a, b, \alpha, \beta):=\bigcup_{c \in \Gamma(a, b)} D(c, \tau) .
$$

Moreover the Lebesgue measure on $R(a, b, \alpha, \beta)$ must be $\beta$-equivalent to the Lebesgue measure on the product $(-\tau, \tau) \times \Gamma(a, b)$, which means that, for any positive continuous function $f$ on $R(a, b, \alpha, \beta)$, we have for 
a smooth parametrization $f(z)=\tilde{f}(t, c)$ of $R(a, b, \alpha, \beta)$

$$
\begin{aligned}
\frac{1}{\beta} \int_{-\tau}^{\tau} \int_{\Gamma(a, b)} \tilde{f}(t, c) d c d t & \leq \int_{R(a, b, \alpha, \beta)} f(z) d m(z) \\
& \leq \beta \int_{-\tau}^{\tau} \int_{\Gamma(a, b)} \tilde{f}(t, c) d c d t
\end{aligned}
$$

Lemma 3.5. Let $(A, B)$ be a restricted or a Hoffman partition of $S$. Then we can make tubes $R(a, \varphi(a), s, \pi / 2)$ which are disjoint.

Proof: If $(A, B)$ is a restricted good partition, then for any $a \in A$ we take the tube of width $r(1-|a|)$ around the segment $(a, \varphi(a))$. Because $a, b:=$ $\varphi(a)$ belong to the same strip $C_{n}(\gamma)$, we have that $\gamma \leq \frac{1-|a|}{1-|\varphi(a)|} \leq 1 / \gamma$. Also $S$ being $\delta$-separated, there is no point of $S$ in a disc $D(b, \delta(1-|b|))$ centered at $b$ and of radius $\delta(1-|b|)$. Moreover, because $b$ is the nearest point in $S$ to $a$, in the hyperbolic metric, the disc $Q(a, b)$ of all the points in $\mathbb{D}$ nearer to $a$ than $b$ contains no other points of $S$; hence the tube with width $s=\min (\delta, r)$ does not contains any point in $S$ but $a$ and $b$. See Figure 3.

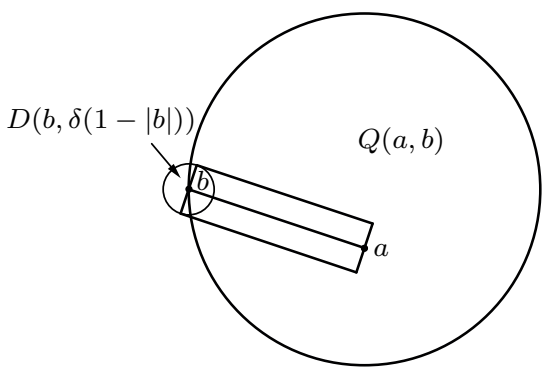

FigURE 3 .

So we take these tubes, which are disjoint, in the case of a restricted good partition.

If $(A, B)$ is a Hoffman partition, again $a$ and $b:=\varphi(a)$ are in the same strip $C_{n}(\gamma)$ hence $1-|a| \simeq 1-|b|$.

Because again the points in $S$ are $\delta$-separated, we can perturb a little bit the segment $(a, b)$ in order to avoid discs $D(c, \delta(1-|c|)), c \in S \backslash\{a, b\}$ with a curve whose length is less than $\pi / 2$ times the length of $(a, b)$. 


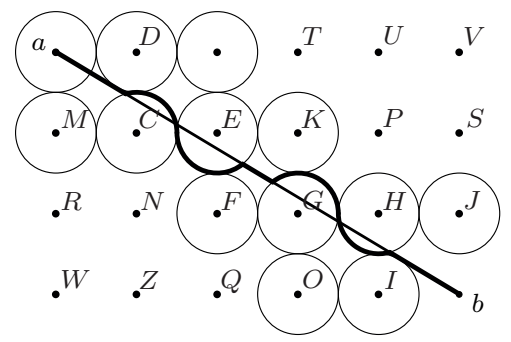

FIGURE 4.

This means that around this curve we can make a tube of width less than $(\delta / 4) \times(1-|a|)$ and these tubes are still disjoint provided that no segments $(a, \varphi(a))$ and $\left(a^{\prime}, \varphi\left(a^{\prime}\right)\right)$ cut each other. But by the construction of the Hoffman partition this cannot happen. We have now to take $s=\min (\delta / 4, r)$ to have that the tubes $R(a, \varphi(a), s, \pi / 2)$ are disjoint.

Because $S$ is $\kappa$-ultra-separated, we have $\exists f \in H^{\infty}(\mathbb{D}):\|f\|_{\infty} \leq 1, \forall a \in A,|f(a)| \leq \tau<\eta^{\kappa}, \forall b \in B,|f(b)| \geq \eta$. Fix $\tau^{\prime}$ satisfying $\tau<\tau^{\prime}<\eta^{\kappa}$, then we have an $r>0$ such that

$$
\forall a \in A, \forall z \in D(a, r(1-|a|)),|f(z)| \leq \tau^{\prime}<\eta^{\kappa} .
$$

By Lemma 3.5 we have that the length of the curve $\Gamma(a, \varphi(a))$ is less than $\pi / 2$ times the length of the segment $(a, \varphi(a))$ so we can enlarge a little bit $W$, say $W^{\prime}:=W^{\prime}(\zeta, \pi h / 2)$, in order to have that

$$
R(a, \varphi(a), s, \pi / 2) \subset W^{\prime} .
$$

See Figure 5.

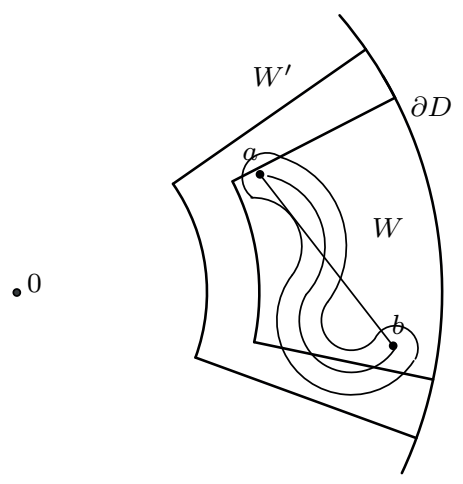

Figure 5. 
The Carleson-Hörmander Lemma 3.4 gives us: there is a $\psi$ with $0 \leq \psi \leq 1$ so that $|\operatorname{grad} \psi| d m$ is a Carleson measure in $\mathbb{D}$ and

$$
\psi(z)=0 \text { when }|f(z)|<\eta^{\kappa}, \quad \psi(z)=1 \text { when }|f(z)|>\eta .
$$

Let $b:=\varphi(a)$ and $I_{a}:=[-s(1-|a|),+s(1-|a|)]$. Parametrize the tube $R(a, \varphi(a), s, \pi / 2)$ :

$$
R(a, \varphi(a), s, \pi / 2)=I_{a} \times \Gamma(a, b) .
$$

Then, because $\psi=0$ on $I_{a} \times\{a\}$ and $\psi=1$ on $I_{a} \times\{b\}$, by the known facts 1 and 2 and the construction of the $R(a, \varphi(a), s, \pi / 2)$, we have $\forall t \in I_{a}, 1=\psi(t, b)-\psi(t, a)=\int_{\Gamma(a, b)} \operatorname{grad} \psi \cdot d s \Rightarrow 1 \leq \int_{\Gamma(a, b)}|\operatorname{grad} \psi| d s$.

Now we integrate with respect to $t$ :

$2 s(1-|a|)=\int_{I_{a}} 1 d t \leq \int_{I_{a}} \int_{a}^{b}|\operatorname{grad} \psi| d s d t \leq \frac{\pi}{2} \int_{R(a, b, s, \pi / 2)}|\operatorname{grad} \psi| d m$, because the Lebesgue measure on $R(a, b, s, \pi / 2)$ is $\pi / 2$ equivalent to the product measure.

This gives the estimate for the points $a$ in $W$ such that $R(a, b, s, \pi / 2)$ is in $W^{\prime}=W(\zeta, \pi h / 2)$, because

$$
\begin{aligned}
\sum_{a \in F_{W}}(1-|a|) & \leq \frac{1}{2 s} \sum_{a \in F_{W}} \frac{\pi}{2} \int_{R(a, b, \ldots)}|\operatorname{grad} \psi(z)| d m(z) \\
& \leq \frac{\pi}{4 s} \int_{W^{\prime}}|\operatorname{grad} \psi(z)| d m(z) \leq \frac{\pi^{2}}{8 s} C h,
\end{aligned}
$$

the tubes being disjoint by Lemma 3.5 and the last inequality because $|\operatorname{grad} \psi(z)| d m(z)$ is a Carleson measure.

Hence the sequence $A$ is Carleson and separated so it is $H^{\infty}(\mathbb{D})$-interpolating.

For the sequence $B$ we proceed analogously and we get that $B$ is also separated and Carleson, hence $H^{\infty}(\mathbb{D})$-interpolating. Because the union $S=A \cup B$ is separated, we get that $S$ is still $H^{\infty}(\mathbb{D})$-interpolating and this finishes the proof of the direct part of the theorem.

For the converse part of the theorem let $S$ be an interpolating sequence for $H^{\infty}(\mathbb{D})$. Then $S$ is $\delta$-separated, hence discrete, so take any restricted or Hoffman partition $(A, B)$ of $S$. Because $S$ is $H^{\infty}(\mathbb{D})$-interpolating, there is an $f \in H^{\infty}(\mathbb{D})$ such that $f=0$ on $A, f=1$ on $B$, and $\|f\|_{\infty} \leq$ $C$. This means that $g:=f / C$ ultra-separates the sequence $S$ for any $\kappa>1$.

Now the answer to the question by A. Hartmann: 
Corollary 3.6. Let $S=A \cup B$, where $(A, B)$ is a restricted or a Hoffman partition of $S$. Suppose that the Blaschke product $B_{A}$, precisely zero on $A$, satisfies $\inf _{b \in B}\left|B_{A}(b)\right| \geq \eta>0$. Then $S$ is an $H^{\infty}(\mathbb{D})$ interpolating sequence.

Proof: We have that $0<\eta^{\kappa}$, where $\kappa$ is the Carleson constant, so we can apply Theorem 1.9.

\section{References}

[1] L. Carleson, An interpolation problem for bounded analytic functions, Amer. J. Math. 80 (1958), 921-930.

[2] J. B. Garnett, "Bounded analytic functions", Pure and Applied Mathematics 96, Academic Press, Inc. [Harcourt Brace Jovanovich, Publishers], New York-London, 1981.

[3] A. Hartmann, Extremely weak interpolation in $H^{\infty}$, Proc. Amer. Math. Soc. 140(7) (2012), 2411-2416. DOI : 10.1090/S0002-99392011-10851-7.

[4] L. Hörmander, Generators for some rings of analytic functions, Bull. Amer. Math. Soc. 73 (1967), 943-949. DOI: 10.1090/s00029904-1967-11860-3.

[5] D. Sarroste, Suites d'interpolation, C. R. Acad. Sci. Paris Sér. AB 274 (1972), A1905-A1908.

Institut de Mathématiques de Bordeaux

Université Bordeaux 1

351, cours de la Libération

F 33405 Talence cedex

France

E-mail address: Eric.Amar@math.u-bordeaux1.fr

Primera versió rebuda el 9 d'abril de 2013, darrera versió rebuda el 2 de juny de 2014. 